\title{
On uniform estimate in Calabi-Yau theorem
}

\author{
Zbigniew Błocki \\ Jagiellonian University, Institute of Mathematics, Reymonta 4, 30-059 Kraków, Poland \\ (email: blocki@im.uj.edu.pl) \\ Received December 10, 2004
}

Abstract We show that the uniform estimate in the Calabi-Yau theorem easily follows from the local stability of the complex Monge-Ampère equation.

Keywords: Kähler manifold, Calabi-Yau theorem.

DOI: $10.1360 / 05 z a 0018$

\section{Introduction}

Let $(M, \omega)$ be a compact Kähler manifold of the complex dimension $n$. In his celebrated paper ${ }^{[1]}$ Yau proved that for any $f \in C^{\infty}(M), f>0$, satisfying the necessary condition

$$
\int_{M} f \omega^{n}=\int_{M} \omega^{n}
$$

there exists, unique up to a constant, solution of the following Dirichlet problem for the complex Monge-Ampère equation on $M$

$$
\left\{\begin{array}{l}
\varphi \in C^{\infty}(M), \\
\omega+i \partial \bar{\partial} \varphi>0, \\
(\omega+i \partial \bar{\partial} \varphi)^{n}=f \omega^{n} .
\end{array}\right.
$$

This gave the affirmative answer to the Calabi conjecture.

By the continuity method and standard Schauder theory one can reduce the proof of the Calabi-Yau theorem to the a priori estimate for solutions of (1)

$$
\|\varphi\|_{C^{2, \alpha}(M)} \leqslant C
$$

where $C>0$ and $\alpha \in(0,1)$ depend only on $M$ and $f$. One of the main difficulties in establishing (2) turned out to be the uniform estimate for the normalized solutions (for example by $\max _{M} \varphi=0$ )

$$
\|\varphi\|_{L^{\infty}(M)} \leqslant C \text {. }
$$

This is contrary to the Dirichlet problem for the complex Monge-Ampère equation on bounded domains in $\mathbb{C}^{n}$, where the uniform estimate follows trivially from the comparison principle ${ }^{[2,3]}$.

The original Yau's proof of the uniform estimate was rather complicated and was subsequently simplified in ref. [4] (see also ref. [5], p. 91 and ref. [6], p. 49).

Copyright by Science in China Press 2005 
A detailed historical account can be found in ref. [5], p. 115. A different proof was given by Kołodziej ${ }^{[7]}$ (see also refs. $[8,9]$ ), where the pluripotential theory was used, one of the main tools being the Bedford-Taylor capacity defined in ref. [10].

The aim of this note is to show that the uniform estimate in the CalabiYau theorem can be very easily deduced from the local stability of the complex Monge-Ampère equation. Since the $L^{2}$ stability can be showed quite easily, we obtain a very simple proof of the uniform estimate.

\section{The $L^{2}$ stability}

The main tool we will use is the following $L^{2}$ stability for the complex Monge-Ampère equation. It was originally established by Cheng and Yau (see ref. [11], p. 75). The Cheng-Yau argument was made precise by Cegrell and Persson $^{[12]}$.

Theorem 1. Let $\Omega$ be a bounded domain in $\mathbb{C}^{n}$. Assume that $u \in C(\bar{\Omega})$ is plurisubharmonic and $C^{2}$ in $\Omega, u=0$ on $\partial \Omega$, and set $f:=\operatorname{det}\left(u_{j \bar{k}}\right)$ (we use the notation $u_{j}=\partial u / \partial z_{j}, u_{\bar{j}}=\partial u / \partial \bar{z}_{j}$ etc.). Then

$$
\|u\|_{L^{\infty}(\Omega)} \leqslant c_{n} \operatorname{diam}(\Omega)\|f\|_{L^{2}(\Omega)}^{1 / n},
$$

where $c_{n}>0$ depends only on $n$.

We will in fact only need the following consequence.

Corollary 2. If $\Omega, u, f$ and $c_{n}$ are as in Theorem 1 , then

$$
\|u\|_{L^{\infty}(\Omega)} \leqslant c_{n} \operatorname{diam}(\Omega)(\operatorname{vol}(\Omega))^{1 / 2 n}\|f\|_{L^{\infty}(\Omega)}^{1 / n} .
$$

Note that by the comparison principle one can easily obtain the above estimate without the dependence on the volume of $\Omega$. For the convenience of the reader, we are now going to sketch the proof of Theorem 1.

Proof of Theorem 1. We use the theory of convex functions and the real Monge-Ampère operator. From ref. [13], Lemma 9.2 we get

$$
\|u\|_{L^{\infty}(\Omega)} \leqslant \frac{\operatorname{diam}(\Omega)}{\lambda_{2 n}^{1 / 2 n}}\left(\int_{\Gamma} \operatorname{det} D^{2} u\right)^{1 / 2 n},
$$

where $\lambda_{2 n}=\pi^{n} / n$ ! is the volume of the unit ball in $\mathbb{C}^{n}$ and

$$
\Gamma:=\{x \in \Omega: u(x)+\langle D u(x), y-x\rangle \leqslant u(y) \forall y \in \Omega\} \subset\left\{D^{2} u \geqslant 0\right\} .
$$

If $w^{1}, \cdots, w^{n}$ are the unit eigenvectors of $\left(u_{j \bar{k}}\right)$ in $\mathbb{C}^{n}$, then $w^{1}, \cdots, w^{n}, i w^{1}, \cdots, i w^{n}$ form an orthonormal basis in $\mathbb{R}^{2 n}$ and at a point where $D^{2} u \geqslant 0$ we obtain

$$
\begin{aligned}
\operatorname{det}\left(u_{j \bar{k}}\right) & =\prod_{l=1}^{n} \sum_{j, k=1}^{n} u_{j \bar{k}} w_{j}^{l} \overline{w_{k}^{l}} \\
& =4^{-n} \prod_{l=1}^{n} \sum_{j, k=1}^{n}\left(D^{2} u \cdot\left(w^{l}\right)^{2}+D^{2} u \cdot\left(i w^{l}\right)^{2}\right)
\end{aligned}
$$




$$
\begin{aligned}
& \geqslant 2^{-n} \sqrt{\prod_{l=1}^{n}\left(D^{2} u \cdot\left(w^{l}\right)^{2}\right)\left(D^{2} u \cdot\left(i w^{l}\right)^{2}\right)} \\
& \geqslant 2^{-n} \sqrt{\operatorname{det} D^{2} u}
\end{aligned}
$$

(the last inequality follows because for real nonnegative symmetric matrices $\left(a_{p q}\right)$ one has $\left.\operatorname{det}\left(a_{p q}\right) \leqslant a_{11} \cdots a_{m m}\right)$. We get the theorem with $c_{n}=2(n !)^{1 / 2 n} / \sqrt{\pi}$.

\section{The uniform estimate}

The uniform estimate will easily follow from the next result.

Proposition 3. Let $\Omega$ be a bounded domain in $\mathbb{C}^{n}$ and $u$ a negative $C^{2}$ plurisubharmonic function in $\Omega$. Assume that $a>0$ is such that the set $\left\{u<\inf _{\Omega} u+a\right\}$ is nonempty and relatively compact in $\Omega$. Then

$$
\|u\|_{L^{\infty}(\Omega)} \leqslant a+\left(c_{n} \operatorname{diam}(\Omega) / a\right)^{2 n}\|u\|_{L^{1}(\Omega)}\|f\|_{L^{\infty}(\Omega)}^{2},
$$

where $f:=\operatorname{det}\left(u_{j \bar{k}}\right)$ and $c_{n}$ is the constant from Theorem 1 .

Proof. Set $t:=\inf _{\Omega} u+a, v:=u-t$ and $\Omega^{\prime}:=\{v<0\}$. By Corollary 2

On the other hand,

$$
a=\|v\|_{L^{\infty}\left(\Omega^{\prime}\right)} \leqslant c_{n} \operatorname{diam}\left(\Omega^{\prime}\right)\left(\operatorname{vol}\left(\Omega^{\prime}\right)\right)^{1 / 2 n}\|f\|_{L^{\infty}\left(\Omega^{\prime}\right)}^{1 / n} .
$$

and the estimate follows.

$$
\operatorname{vol}\left(\Omega^{\prime}\right) \leqslant \frac{\|u\|_{L^{1}(\Omega)}}{|t|}=\frac{\|u\|_{L^{1}(\Omega)}}{\|u\|_{L^{\infty}(\Omega)}-a}
$$

We are now in position to prove the uniform estimate.

Theorem 4. Let $(M, \omega)$ be the compact Kähler manifold of dimension $n$. Assume that $\varphi \in C^{2}(M)$ is such that $\max _{M} \varphi=0, \omega+i \partial \bar{\partial} \varphi \geqslant 0$ and $(\omega+i \partial \bar{\partial} \varphi)^{n}=f \omega^{n}$. Then

$$
\|\varphi\|_{L^{\infty}(M)} \leqslant C
$$

where $C>0$ depends only on $M$ and on an upper bound for $\|f\|_{L^{\infty}(M)}$.

Proof. From $\omega+i \partial \bar{\partial} \varphi \geqslant 0$ it follows in particular that $\Delta \varphi \geqslant-n / 2$ and using the Green function for the Laplace-Beltrami operator on compact Riemannian manifolds (see e.g. ref. [1]) in the standard way we obtain

$$
\|\varphi\|_{L^{1}(M)} \leqslant C(M) \text {. }
$$

Let $z_{0} \in M$ be such that $\varphi\left(z_{0}\right)=\min _{M} \varphi$. We can find $U$, a chart containing $z_{0}$, and a $C^{\infty}$ smooth, strongly plurisubharmonic function $g$ in $U$ with $\omega=i \partial \bar{\partial} g$. The Taylor expansion of $g$ about $z_{0}$ gives

where

$$
\begin{aligned}
g\left(z_{0}+h\right) & =\operatorname{Re} P(h)+2 \sum_{j, k=1}^{n} g_{j \bar{k}}\left(z_{0}\right) h_{j} \bar{h}_{k}+\frac{1}{3 !} D^{3} g(\widetilde{z}) \cdot h^{3} \\
& \geqslant \operatorname{Re} P(h)+c_{1}|h|^{2}-c_{2}|h|^{3},
\end{aligned}
$$

$$
P(h)=g\left(z_{0}\right)+2 \sum_{j} g_{j}\left(z_{0}\right) h_{j}+2 \sum_{j, k} g_{j k}\left(z_{0}\right) h_{j} h_{k}
$$


is a complex polynomial (and thus $i \partial \bar{\partial}(\operatorname{Re} P)=0$ ), $\widetilde{z} \in\left[z_{0}, z_{0}+h\right]$ and $c_{1}, c_{2}>0$ depend only on $M$. Replacing $g$ with $g$ - Re $P$-const. (which does not change the Kähler form $\omega$ ) we may thus assume that there exist $a, r>0$ depending only on $M$ such that $g<0$ in $B\left(z_{0}, 2 r\right), g$ attains minimum in $B\left(z_{0}, 2 r\right)$ at $z_{0}$ and $g \geqslant g\left(z_{0}\right)+a$ on $B\left(z_{0}, 2 r\right) \backslash B\left(z_{0}, r\right)$. Now Proposition 3 for $\Omega:=B\left(z_{0}, 2 r\right)$ and $u:=g+\varphi$ combined with (3) gives the required estimate.

Remark. Using the Hölder inequality in Corollary 2 we will get for every $p>2$,

$$
\|u\|_{L^{\infty}(\Omega)} \leqslant c_{n} \operatorname{diam}(\Omega)(\operatorname{vol}(\Omega))^{1 / 2 q n}\|f\|_{L^{p}(\Omega)}^{1 / n},
$$

where $q$ is such that $\frac{2}{p}+\frac{1}{q}=1$. Therefore, we can replace the $L^{\infty}$ norm of $f$ in Theorem 4 by the $L^{p}$ norm for any $p>2$. Moreover, since Kolodziej ${ }^{[14]}$ showed (with more complicated proof) that the $L^{p}$ stability for the complex MongeAmpère equation holds for every $p>1$ (that is the $L^{2}$ norm of $f$ in Theorem 1 can be replaced by the $L^{p}$ norm, and even by a weaker Orlicz norm), we can do this for every $p>1$ (and even for the Orlicz norm introduced by Kołodziej). This was shown in ref. [7], where the local techniques from ref. [14] had to be repeated on $M$. Our argument shows that the global uniform estimate in fact follows easily from the local results.

Acknowledgements The author is grateful to the organizers of the 2004 Beijing International Conference on Several Complex Variables for the invitation.

\section{References}

1. Yau, S. -T., On the Ricci curvature of a compact Kähler manifold and the complex Monge-Ampère equation, I, Comm. Pure Appl. Math., 1978, 31: 339-411.

2. Bedford, E., Taylor, B. A., The Dirichlet problem for a complex Monge-Ampère equation, Invent. Math., 1976, 37: 1-44.

3. Caffarelli, L., Kohn, J. J., Nirenberg, L. et al., The Dirichlet problem for non-linear second order elliptic equations II: Complex Monge-Ampère, and uniformly elliptic equations, Comm. Pure Appl. Math., 1985, 38: 209-252.

4. Kazdan, J. L., A remark on the proceding paper of Yau, Comm. Pure Appl. Math., 1978, 31: $413-414$

5. Siu, Y. -T., Lectures on Hermitian-Einstein Metrics for Stable Bundles and Kähler-Einstein Metrics, Boston: Birkhäuser, 1987.

6. Tian, G., Canonical Metrics in Kähler Geometry, Boston: Birkhäuser, 2000.

7. Kołodziej, S., The complex Monge-Ampère equation, Acta Math., 1998, 180: 69—117.

8. Kołodziej, S., The Complex Monge-Ampère Equation and Pluripotential Theory, Memoirs Amer. Math. Soc., to appear.

9. Tian, G., Zhu, X., Uniqueness of Kähler-Ricci solitons, Acta Math., 2000, 184: 271-305.

10. Bedford, E., Taylor, B. A., A new capacity for plurisubharmonic functions, Acta Math., 1982, 149: $1-41$.

11. Bedford, E., Survey of pluri-potential theory, in Several Complex Variables, Proceedings of the Mittag-Leffler Institute, 1987-1988 (ed. Fornæss, J. E.), Princeton: Princeton Univ. Press, 1993.

12. Cegrell, U., Persson, L., The Dirichlet problem for the complex Monge-Ampère operator: Stability in $L^{2}$, Michigan Math. J., 1992, 39: 145-151.

13. Gilbarg, D., Trudinger, N. S., Elliptic Partial Differential Equations of Second Order, Classics in Mathematics, Berlin: Springer-Verlag, 1998.

14. Kołodziej, S., Some sufficient conditions for solvability of the Dirichlet problem for the complex Monge-Ampère operator, Ann. Pol. Math., 1996, 65: 11-21. 